AN. MED. INTERNA (Madrid) Vol. 24 , N. ${ }^{\circ} 10$, pp. $478-483,2007$

\title{
Análisis y factores predictores del retraso en la sospecha diagnóstica y tratamiento de los pacientes hospitalizados con tuberculosis pulmonar
}

\author{
J. L. MOSTAZA, N. GARCÍA ${ }^{1}$, S. FERNÁNDEZ ${ }^{1}$, A. BAHAMONDE ${ }^{1}$, \\ M. I. FUENTES ${ }^{1}$, M. J. PALOMO ${ }^{1}$ \\ Servicio de Medicina Interna. Complejo Asistencial de León. ${ }^{1}$ Hospital del Bierzo. \\ Fuentesnuevas. Ponferrada, León
}

\begin{abstract}
ANALYSIS AND PREDICTORS OF DELAYS IN THE SUSPICION AND TREATMENT AMONG HOSPITALIZED PATIENTS WITH PULMO-
\end{abstract} NARY TUBERCULOSIS

\section{RESUMEN}

Fundamento y objetivo: en algunos pacientes el diagnóstico de tuberculosis no es sospechado en el momento del ingreso hospitalario, favoreciendo su transmisión nosocomial. El objetivo del estudio era valorar el retraso diagnóstico que se produce en los pacientes ingresados con tuberculosis respiratoria en nuestro hospital y los factores que lo determinan.

Pacientes y métodos: estudio retrospectivo de todos los pacientes hospitalizados con tuberculosis pulmonar entre 1995 y 2002. En cada paciente se registraron variables clínico-epidemiológicas y el tiempo desde el ingreso hasta el inicio del aislamiento respiratorio y del tratamiento antituberculoso.

Resultados: 149 pacientes fueron diagnosticados de tuberculosis pulmonar durante una hospitalización. El diagnóstico no fue sospechado en las primeras 24 horas del ingreso en 102 pacientes (68\% [IC95\%, 61 a $75 \%])$, de los cuales $66(64,7 \%$ [IC95\%, 56 a 74\%]) tenían tinción de esputo positiva. En 62 pacientes (42\% [IC95\%, 34 a 50\%]) se inició tratamiento tuberculostático pasados más de 7 días desde el ingreso, de ellos 34 (55\% [IC95\%, 43 a 67\%]) tenían tinción de esputo positiva. Se asocian independientemente a mayor retraso diagnóstico: edad $\geq 60$ años (OR 3,17 [IC95\%; 1,27 a 7,87]; p = 0,013), presencia de enfermedad pulmonar crónica (OR 2,99 [IC95\%; 1,21 a 7,38]; p = 0,017), tinción de esputo negativa (OR 4,51 [IC95\%; 1,34 a 15,16]; $\mathrm{p}=0,015)$ y ausencia de hemoptisis o melanoptisis (OR 2,96 [IC95\%; 1,18 a 7,41]; $\mathrm{p}=0,020$ ).

Conclusiones: El retraso en el diagnóstico y tratamiento de pacientes que ingresan con tuberculosis respiratoria es frecuente, incluso en pacientes con tinción de esputo positiva, por falta de sospecha clínica (sobre todo en pacientes ancianos, con patología pulmonar crónica o con manifestaciones atípicas de la enfermedad). Estos datos ponen de manifiesto la necesidad de mantener un alto índice de sospecha, desarrollar pruebas de diagnóstico rápido e iniciar precozmente un tratamiento empírico para mejorar el control de la enfermedad.

PALABRAS CLAVE: Diagnóstico de tuberculosis. Tratamiento de tuberculosis. Control de tuberculosis. Diagnóstico retrasado. Transmisión nosocomial.

\section{ABSTRACT}

Background and objectives: Sometimes tuberculosis diagnosis is missed at hospital admission. Delayed diagnosis of active pulmonary tuberculosis among hospitalized patients could to contribute to nosocomial transmission. The objective of this study was to define the occurrence and associated patient risk factors among hospitalized patients with delayed diagnosis of respiratory tuberculosis.

Methods and patients: A retrospective chart review was undertaken between 1995 and 2002 on all patients with pulmonary tuberculosis. Time intervals between admission, diagnosis and treatment of tuberculosis were determined. Epidemiological and clinical features were evaluated for their effect on these time intervals.

Results: Among 149 patients newly diagnosed to have active pulmonary $T B$, the diagnosis was initially missed in $102(68 \%$ [95\% CI, 61 to $75 \%])$ of all hospitalized patients, of whom 66 (65\% [95\% CI, 56 to $74 \%]$ ) were smear positive. Treatment was initiated after a week or more in $62(42 \%$ [95\% CI, 34\% to 50\%]) of all patients, of whom $34(55 \%$ [95\% CI, 43 to 67\%])) were smear positive. Age $\geq 60$ years (OR 3.17 [95\% CI; 1.27 to 7.87$] ; p=0.013$ ), presence of chronic lung disease (OR 2.99 [95\% CI; 1.21 to 7.38]; $p=0.017)$, negative sputum AFB smear (OR 4.51 [95\% CI; 1.34 to 15.16]; $p=0.015)$ and absence of hemoptysis or melanoptysis (OR 2.96 [95\% CI; 1.18 a 7.41]; $p=0.020$ ), were independently associated with delays.

Conclusions: The diagnosis and treatment of hospitalized patients with pulmonary tuberculosis is often delayed because absence of clinic suspicion owing to old age, chronic lung disease or atypical presentations and slow confirmation by culture. Improved clinical acumen, development of rapid diagnostic tests, and the institution of early empiric therapy are desirable objectives to improve the tuberculosis control.

KEY WORDS: Tuberculosis diagnosis. Tuberculosis treatment. Tuberculosis control. Delayed diagnosis. Nosocomial transmission.

Mostaza JL, García N, Fernández S, Bahamonde A, Fuentes MI, Palomo MJ. Análisis y factores del retraso en la sospecha diagnóstica y tratamiento de los pacientes hospitalizados con tuberculosis pulmonar. An Med Interna (Madrid) 2007; 24: 478-483.

\section{INTRODUCCIÓN}

El mantenimiento de un alto índice de sospecha, el aislamiento respiratorio precoz y la rápida iniciación del trata- miento, son los puntos clave para evitar la propagación nosocomial de la tuberculosis (1-4). El retraso en el diagnóstico de pacientes hospitalizados con tuberculosis activa puede contribuir a una mayor morbi-mortalidad y a un aumento en su

Trabajo aceptado: 25 de junio de 2007 
transmisión nosocomial (5-8). Aunque se debería evitar el ingreso de pacientes con tuberculosis respiratoria (salvo casos graves, presencia de complicaciones de la enfermedad o por imposibilidad de atención o aislamiento en el domicilio), la realidad es que ingresan un porcentaje significativo de enfermos con tuberculosis, por distintas razones, entre ellas, la falta de sospecha clínica en el momento del ingreso (9-13).

La extensión y las causas del retraso diagnóstico en pacientes ingresados con tuberculosis son poco conocidas y pueden variar de un hospital a otro, pero podrían achacarse a alguno de los siguientes motivos: manifestaciones atípicas de la enfermedad, escasa pericia del médico para la sospecha clínica o falta de diligencia del laboratorio en el análisis y comunicación de los resultados positivos.

El objetivo del estudio era analizar, en nuestro medio, el retraso diagnóstico de los pacientes hospitalizados con tuberculosis respiratoria y determinar las características clínicas asociadas a un mayor retraso diagnóstico.

\section{MATERIAL Y MÉTODOS}

-Contexto: El Hospital del Bierzo tiene 350 camas y está situado en Ponferrada, en el noroeste de la provincia de León. Da servicio a una comarca de 150.000 habitantes, en la que la minería ha sido la actividad económica principal, condicionando una prevalencia elevada de neumoconiosis y alta incidencia de tuberculosis $(13,14)$.

-Pacientes: estudio retrospectivo de los pacientes hospitalizados con tuberculosis pulmonar entre 1995 y 2002.

- Medidas: En cada paciente se registraron los datos demográficos, así como las características clínicas y radiológicas en el momento de la solicitud de estudio de micobacterias. Las variables del estudio fueron: edad, sexo, profesión, presencia de comorbilidad, fiebre, síntomas respiratorios, hallazgos radiológicos en la placa de tórax y positividad de la tinción de esputo para micobacterias. También se registró el tiempo desde el ingreso hasta el inicio del aislamiento respiratorio y del tratamiento antituberculoso.

-Definiciones: Retraso en la sospecha diagnóstica (RD): número de días transcurridos desde el ingreso hasta la fecha en que se ordena el aislamiento respiratorio o se inicia el tratamiento antituberculoso. Retraso en el tratamiento (RT): número de días transcurridos desde el ingreso hasta el inicio del tratamiento antituberculoso. Diagnóstico no sospechado al ingreso (DNSI): si no se inició el aislamiento respiratorio o el tratamiento antituberculoso en las primeras $24 \mathrm{~h}$ del ingreso. Manifestaciones radiológicas atípicas (MRA): presencia de infiltrados en zonas pulmonares declives o ausencia de modificaciones radiológicas respecto a las placas previas.

- Análisis de los datos: En el estudio bivariado, las diferencias entre los grupos se analizaron utilizando para las variables categóricas la prueba de $\chi^{2}$ y la prueba exacta de Fisher; para el análisis de las variables continuas se utilizaron la prueba $t$ de Student si seguían una distribución normal y la prueba de Mann-Whitney (Wilcoxon) para las distribuciones no normales. Se realizó un análisis multivariante de regresión logística para analizar las asociaciones significativas entre las variables clínico-demográficas y DNSI en el análisis univariante, con cálculo de la odds-ratio y el intervalo de confianza del $95 \%$. Se consideraron diferencias significativas entre los grupos, si el valor de $p$ era menor de 0,05 .

\section{RESULTADOS}

Entre los años 1995 y 2002 se diagnosticó de tuberculosis pulmonar a 261 pacientes. En 150 pacientes, el diagnóstico de tuberculosis pulmonar se realizó durante un ingreso hospitalario, de los cuales se pudieron analizar las historias de 149 pacientes, que representan el $0,15 \%$ sobre el total de pacientes ingresados en el hospital.

En los pacientes ingresados con tuberculosis pulmonar activa la mediana de edad era de 58 años (rango intercuartílico: 3073 años) y 111 eran varones $(74,5 \%) ; 109$ pacientes $(73,15 \%)$ tenían baciloscopia positiva y 32 pacientes $(21,48 \%)$ tenían localización extrapulmonar simultánea. La tabla I muestra las características clínicas y epidemiológicas de los pacientes ingresados con tuberculosis pulmonar.

TABLA I

\section{VARIABLES CLÍNICAS EPIDEMIOLÓGICAS}

\begin{tabular}{lcc}
\hline & $N$ & $\%$ \\
\hline Edad $\geq 60$ a. & 64 & 42,95 \\
Hombres & 111 & 74,5 \\
Mineros & 52 & 34,9 \\
Infección por VIH & 12 & 8,05 \\
Localización pulmonar y extrapulmonar & 32 & 21,48 \\
Pat. pulmonar crónica & 63 & 42,28 \\
Neumoconiosis & 36 & 24,16 \\
Fiebre & 81 & 54,36 \\
Tos & 117 & 78,52 \\
Hemoptisis & 29 & 19,46 \\
Melanoptisis & 11 & 7,38 \\
Tinción de esputo positiva & 109 & 73,15 \\
Manifestaciones radiológicas atípicas & 44 & 29,53 \\
\hline
\end{tabular}

La mediana del intervalo desde ingreso hasta aislamiento fue de 5 días (rango intercuartílico 1-13) y desde ingreso a tratamiento 5 días (rango intercuartílico 2-12). El diagnóstico de tuberculosis no fue sospechado en el momento del ingreso (DNSI) en 102 pacientes (68\% [IC95\%, 61 a 75\%]), de los cuales 66 (64,7\% [IC95\%, 56 a 74\%]) tenían tinción de esputo positiva. En 62 pacientes (42\% [IC95\%, 34 a 50\%]) se inició tratamiento tuberculostático pasados más de 7 días desde el ingreso, de los cuales 34 (55\% [IC95\%, 43 a 67\%]) tenían tinción de esputo positiva. La tabla II y las figuras 1 y 2 muestran el porcentaje de pacientes con distintos intervalos de RD

\section{TABLA II}

INTERVALOS DE RETRASO

\begin{tabular}{lcccc}
\hline & $n$ & $\%$ & $n$ & $\%$ \\
& \multicolumn{1}{c}{$R D^{*}$} & & & $R T^{*}$ \\
\hline Total & 149 & & & \\
$>$ 1 día & 102 & 68,46 & 115 & 77,18 \\
$>$ 2 días & 96 & 64,43 & 104 & 69,8 \\
$>7$ días & 55 & 36,91 & 62 & 41,6 \\
$>$ 14 días & 25 & 16,78 & 29 & 19,46 \\
$>$ 30 días & 11 & 7 & 13 & 8,72 \\
Mediana (RI)*** & $4(1-11)$ & $5(2-12)$ & & \\
\hline
\end{tabular}

${ }^{*} \mathrm{RD}$ : retraso diagnóstico; ${ }^{*} \mathrm{RT}$ : retraso en tratamiento; ${ }^{*}{ }^{*} \mathrm{RI}$ : rango intercuartílico. 


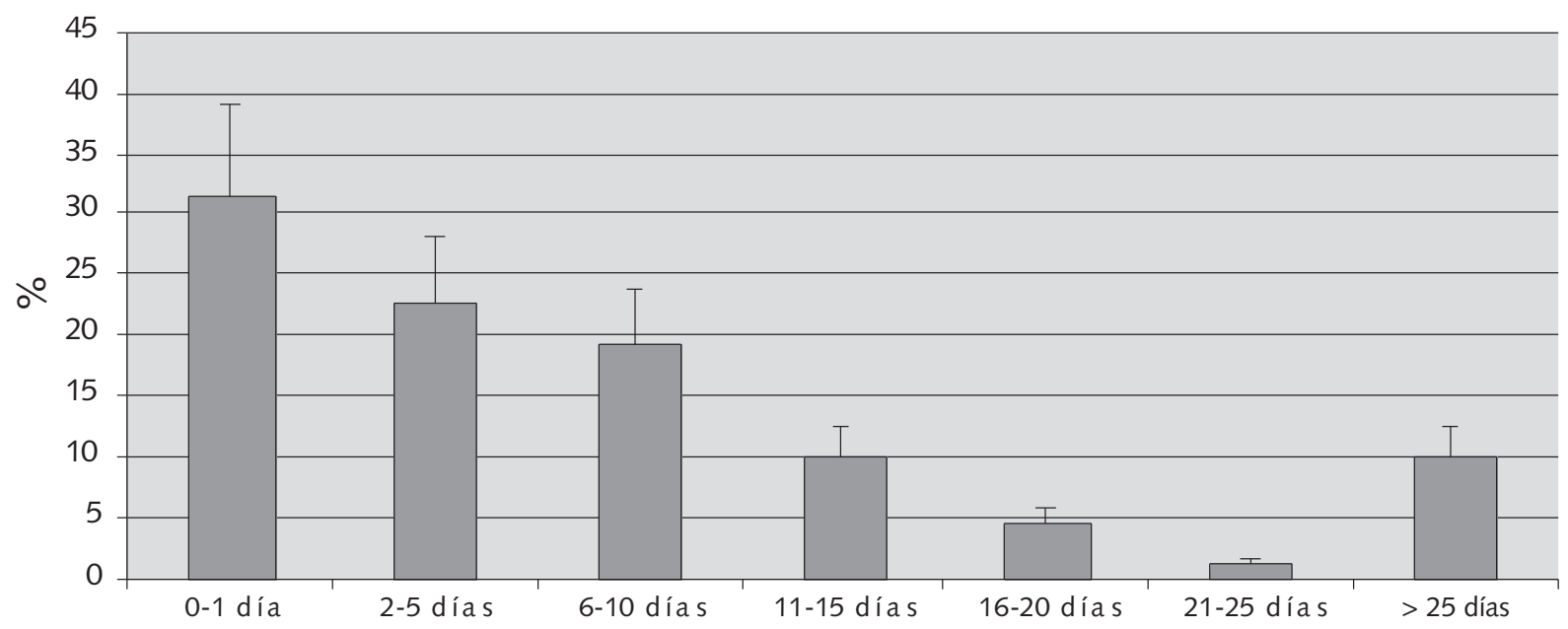

Fig. 1. Distribución de intervalos de retraso diagnóstico. Se muestra el límite superior del IC95\%.

TABLA III

PORCENTAJE DE PACIENTES CON DNSI* (ANÁLISIS UNIVARIANTE)

\begin{tabular}{lccc}
\hline & Si (\%) & No $(\%)$ & $p$ \\
\hline Edad $\geq 60$ años & 84,37 & 56,47 & 0,0003 \\
Sexo varón & 72,07 & 57,84 & 0,1045 \\
Localización tuberculosa múltiple & 84,37 & 64,10 & 0,0288 \\
Patología pulmonar crónica & 80,95 & 59,30 & 0,0050 \\
Neumoconiosis & 83,33 & 63,62 & 0,0274 \\
Infección por VIH & 100 & 65,69 & 0.0142 \\
Fiebre & 61,76 & 74,07 & 0,1073 \\
Tos & 67,52 & 71,87 & 0,63 \\
Hemoptisis o melanoptisis & 46,15 & 76,36 & 0,0005 \\
Baciloscopia de esputo positiva & 60,55 & 90 & 0,0006 \\
Cavitación en radiología de tórax & 39,13 & 74,4 & 0,0008 \\
Manifestaciones radiológicas atípicas & 81,82 & 62,86 & 0,0231 \\
\hline
\end{tabular}

*DNSI: diagnóstico no sospechado al ingreso; Se compara el porcentaje de pacientes con DNSI entre los que tienen (Sí) o no tienen (No) determinadas características epidemiológicas y clínicas.

\section{TABLA IV}

VARIABLES ASOCIADAS CON DNSI* (ANÁLISIS MULTIVARIANTE)

\begin{tabular}{|c|c|c|c|c|}
\hline & $O R$ & IC95\% inf. & IC95\% sup. & $p$ \\
\hline Edad $\geq 60$ años & 3.1653 & 1.2725 & 7.8735 & 0,0132 \\
\hline Patología pulmonar crónica & 2.9924 & 1.2129 & 7.3830 & 0,0174 \\
\hline $\begin{array}{l}\text { Ausencia de hemoptisis o } \\
\text { melanoptisis }\end{array}$ & 2.9617 & 1.1827 & 7.4171 & 0,0204 \\
\hline Tinción de esputo negativa & 4.5136 & 1.3436 & 15.1631 & 0,0148 \\
\hline $\begin{array}{l}\text { Manifestaciones radiológicas } \\
\text { atípicas }\end{array}$ & 2.1145 & 0.7724 & 5.7886 & 0,1450 \\
\hline
\end{tabular}

*DNSI: diagnóstico no sospechado al ingreso.

y RT. En 13 pacientes (9\% [IC95\%, 4 a 14\%]) el RT fue mayor de 30 días. Entre los 109 pacientes con tinción de esputo positiva, $78(71,55 \%)$ tuvieron un RT mayor de 24 horas.

Entre los pacientes con DNSI la mediana del intervalo desde el ingreso hasta el aislamiento respiratorio fue de 8 días (rango intercuartílico 3-16 días), y desde aislamiento hasta tratamiento era cero días (rango intercuartílico 0-0 días).

- Características clínicas y retraso diagnóstico: El análisis univariante (Tabla III) identificó las características epidemiológicas y clínicas que se asociaron significativamente a una mayor probabilidad de DNSI: mayores de 60 años ( $\mathrm{p}=$ 0,0003 ), localización tuberculosa múltiple (pulmonar y extrapulmonar) $(\mathrm{p}=0,0288)$, presencia de patología pulmonar crónica $(p=0,0050)$, neumoconiosis $(p=0,0274)$, infección por VIH $(p=0,0142)$ e indicadores de manifestaciones atípicas de tuberculosis pulmonar, como ausencia de hemoptisis o melanoptisis $(\mathrm{p}=0,0005)$, tinción de esputo negativa $(\mathrm{p}=0,0005)$, ausencia de cavitación en radiología de tórax $(\mathrm{p}=0,0008)$ y manifestaciones radiológicas atípicas (MRA) $(\mathrm{p}=0,0231)$.

En el análisis multivariante (Tabla IV) se asociaron independientemente con DNSI: Edad $\geq 60$ años, presencia de patología pulmonar crónica, ausencia de hemoptisis o melanoptisis y tinción de esputo negativa.

\section{DISCUSIÓN}

En nuestro hospital, el diagnóstico de tuberculosis pulmonar pasa desapercibido con frecuencia en el momento del ingreso hospitalario, lo que condiciona un retraso en el aislamiento y tratamiento de estos pacientes. El diagnóstico de tuberculosis no fue sospechado en el momento del ingreso en el $68,46 \%$ de los pacientes, de los que un $64,7 \%$ tenían baciloscopia positiva. En el 41,6\% de los casos se inició tratamiento tuberculostático pasados 7 o más días desde el ingreso, de los cuales un 54,8\% tenían baciloscopia positiva.

Nuestros hallazgos, en concordancia con los encontrados por otros autores de hospitales norteamericanos $(12,13)$, demuestran un mayor retraso en el diagnóstico y tratamiento de pacientes que ingresan con tuberculosis pulmonar en: ancianos, pacientes con patología pulmonar crónica (en nuestro caso muchos con neumoconiosis) o con manifestaciones atípicas de la enfermedad (ausencia de hemoptisis o melanoptisis, radiología torácica atípica y tinción de esputo negativa).

Un estudio reciente ha encontrado una relación inversa entre la incidencia de pacientes hospitalizados con tuberculosis y el retraso diagnóstico en un hospital (13), que probablemente se corresponde con un mejor conocimiento de las mani- 


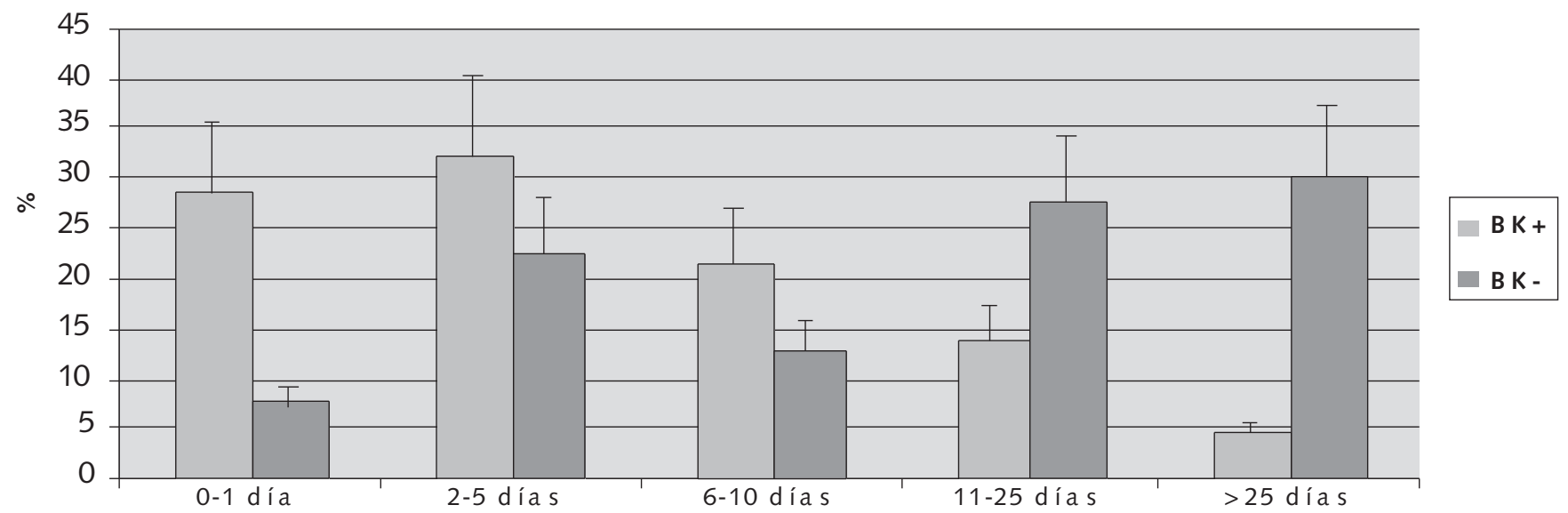

Fig. 2. Porcentaje de pacientes con tinción de esputo positiva ( $B K+)$ y negativa ( $B K-)$ y diferentes intervalos de retraso de tratamiento. Se muestra el límite superior del IC95\%.

TABLA V

COMPARACIÓN DE RESULTADOS CON OTRAS SERIES PUBLICADAS

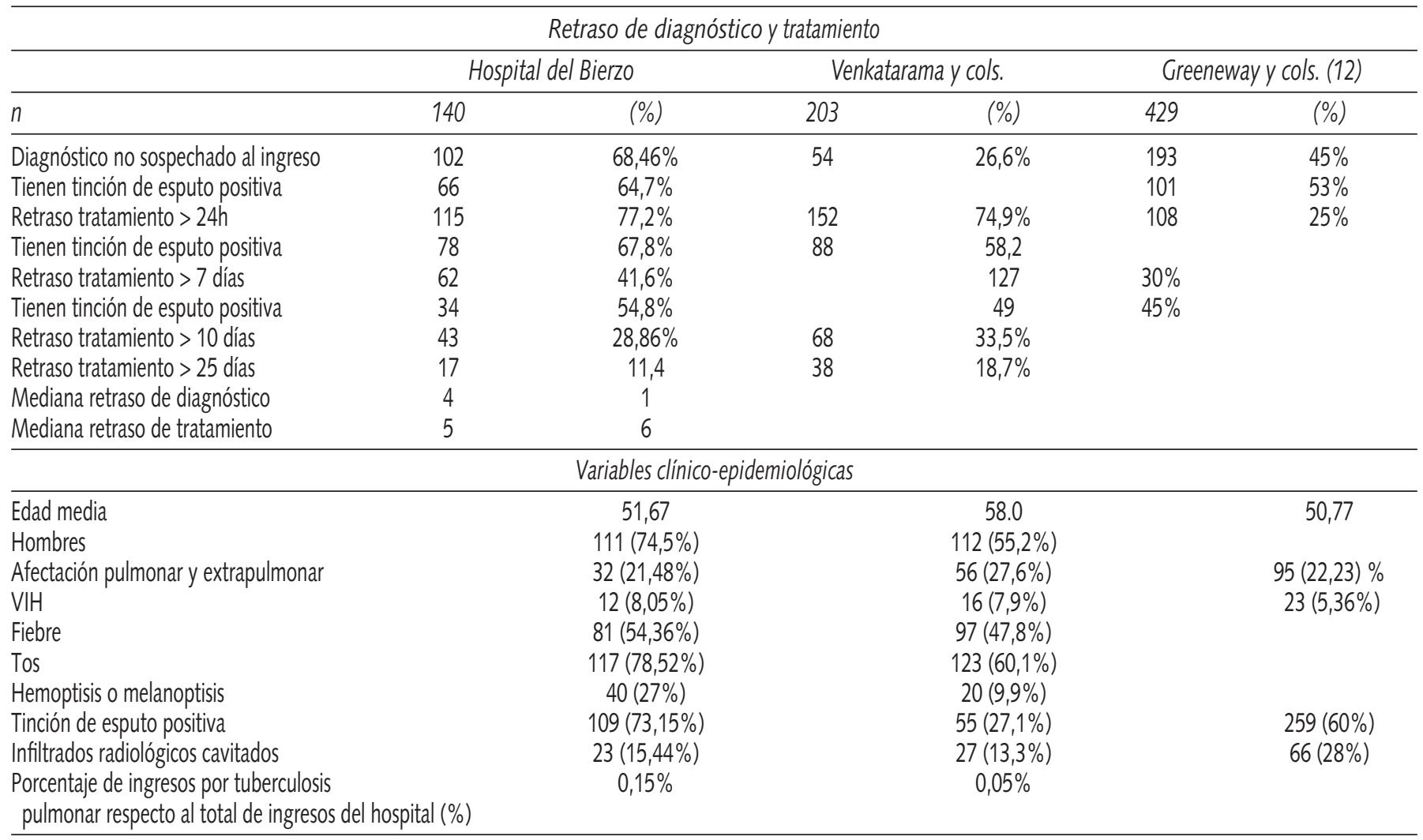

Comparación de variables clínico-epidemiológicas y de resultados de retraso de diagnóstico y tratamiento de tuberculosis durante una hospitalización, con otras series referidas en la bibliografía.

festaciones de la tuberculosis por los médicos de hospitales con mayor incidencia. Nuestro hospital se encuentra en una comarca con elevada incidencia de tuberculosis $(14,15) \mathrm{y}$, sin embargo, hemos encontrado un retraso en el inicio del tratamiento similar o mayor al observado en algunos hospitales norteamericanos localizados en zonas de menor incidencia
$(12,13)$ (Tabla V). Esto podría explicarse, en parte, por la elevada incidencia de neumoconiosis en la serie que presentamos (23,57\% de los pacientes), que como queda reflejado en este estudio se asocia a un mayor retraso diagnóstico. Otro factor que podría haber colaborado al elevado número de pacientes con diagnóstico no sospechado al ingreso es la ausencia de 
microbiólogo de guardia en nuestro hospital, que imposibilita el análisis del esputo fuera de la jornada laboral.

El retraso en el diagnóstico de tuberculosis desde la aparición de los primeros síntomas y las causas que lo generan, ha sido estudiado por diversos autores españoles (16-19). Sin embargo, hasta dónde hemos podido investigar (Medline 1960-2004 y descriptores: "tuberculosis diagnosis", "tuberculosis treatment", "delayed diagnosis" y "nosocomial transmisión"), no hemos encontrado otros estudios de hospitales españoles que analicen el retraso diagnóstico que sufren los pacientes hospitalizados con tuberculosis respiratoria, salvo resultados parciales de este mismo estudio $(20,21)$.

Para estudiar el retraso diagnóstico de pacientes que ingresan con tuberculosis se debe usar un diseño retrospectivo (13), ya que un estudio prospectivo podría diferir de la realidad habitual al elevar la sospecha diagnóstica de tuberculosis entre los médicos participantes del estudio y modificar su comportamiento. No obstante, un estudio retrospectivo tiene limitaciones que dependen en buena medida de lo correcta y exhaustivamente que hayan sido recogidos en la historia los datos clínicos y epidemiológicos que se investigaron. Por otro lado, en algunos pacientes con tuberculosis pulmonar el diagnóstico podría no haberse realizado, si falleció sin practicarse autopsia o fue dado de alta sin investigar la posibilidad de tuberculosis pulmonar. En nuestro estudio puede estar maximizado el retraso hasta aislamiento y hasta diagnóstico, debido a que algunos pacientes podrían haber estado en aislamiento respiratorio sin que figurara en órdenes o curso clínico; el retraso hasta tratamiento es un dato más objetivo al poderse recoger de forma precisa la fecha del ingreso y la de inicio del tratamiento tuberculostático.

A pesar de los avances en las técnicas de cultivo, pasan varias semanas desde la sospecha clínica y recogida de muestras hasta la confirmación microbiológica final. Durante este periodo el paciente no tratado puede empeorar clínicamente y se convierte en una fuente para la diseminación de la enfermedad en la comunidad (o en el hospital, en caso de encontrarse hospitalizado). Aunque los pacientes con baciloscopia de esputo negativa tienen menor riesgo de transmitir la enfermedad, pueden ser responsables de aproximadamente un $17 \%$ de las transmisiones y su impacto sobre la salud pública no debe ser despreciado $(22,23)$.

El aislamiento de todos los pacientes ingresados con sospecha remota de tuberculosis pulmonar puede ser poco práctico y excesivamente caro. Para mejorar la eficiencia del aislamiento respiratorio, se han propuesto varias reglas de predicción clínica en pacientes con sospecha de tuberculosis $(23,24)$.

Moreno et al. (26) nos animan recientemente a no olvidar el riesgo de transmisión nosocomial de la tuberculosis, señalan que es una amenaza real y que nuestras estructuras probablemente no están preparadas para evitarla. Venkatarama y cols. (11), apoyándose en recomendaciones de organismos internacionales $(2,3,31)$, proponen que en todos los pacientes ingresados en el hospital se debería evaluar el riesgo de tuberculosis activa, sobre todo en aquellos hospitales con mayor prevalencia de tuberculosis. La sospecha de tuberculosis debería ser máxima en presencia de lesiones fibróticas en la placa de tórax, neumoconiosis $(27,28)$, infección por $\mathrm{VIH}$ (29) o que están sometidos a tratamiento inmunosupresor (entre los que cabe destacar a los nuevos anti-TNF) (30), que ingresan con síntomas respiratorios, fiebre, síndrome constitucional o se aprecia la presencia de nuevos infiltrados o cavitaciones en la placa de tórax. Los pacientes con sospecha de tuberculosis deben ser sometidos a aislamiento respiratorio y realizar en las primeras 24 horas del ingreso: placa de tórax, prueba de Mantoux y estudio de micobacterias en esputo. El tratamiento antituberculoso se debería iniciar inmediatamente en todos los casos con baciloscopia positiva en esputo o que presenten una placa de tórax con alta sospecha de tuberculosis. El umbral para iniciar tratamiento antituberculoso debe ser bajo en pacientes con características demográficas o clínicas de alto riesgo de tuberculosis. En pacientes de riesgo alto y baciloscopia negativa, se debería mantener el tratamiento hasta que los resultados del cultivo estén disponibles o se haya encontrado un diagnóstico alternativo. El seguimiento de estas recomendaciones puede conducir, en algunos casos, a la iniciación de tratamiento innecesario, pero el medio hospitalario es excesivamente sensible a la difusión de la tuberculosis $(6-8,13)$ y los beneficios de un tratamiento precoz para prevenir la transmisión nosocomial están bien establecidos (1-4,32,33). Por lo tanto, es prudente aplicar una política conservadora para minimizar el riesgo de transmisión nosocomial, al menos hasta que dispongamos de medios diagnósticos más rápidos y precisos.

Este estudio refleja la realidad de nuestro hospital y la situación podría ser diferente en otras zonas. Creemos que la trascendencia del problema justifica la realización de estudios similares en otros hospitales, que pongan de manifiesto la realidad española acerca del retraso diagnóstico intrahospitalario en los pacientes que ingresan con tuberculosis, de cara a planificar las intervenciones necesarias para minimizarlo.

En resumen, hemos encontrado que el diagnóstico de tuberculosis pulmonar en pacientes hospitalizados puede pasar desapercibido con frecuencia, sobre todo en ancianos, pacientes con patología pulmonar crónica o con manifestaciones atípicas de la enfermedad. Esto supone un riesgo para la evolución del propio paciente y una amenaza para la salud del resto de pacientes hospitalizados y del personal sanitario. Mejorar la conciencia del problema de la tuberculosis entre los médicos que atienden a estos pacientes, profundizar en el estudio y conocimiento de las causas que contribuyen a un diagnóstico retrasado y desarrollar nuevas técnicas, más sensibles y rápidas, para el diagnóstico de la tuberculosis, es el reto al que nos enfrentamos.

\section{AGRADECIMIENTOS}

A los doctores R. López y C. Madroñero, por la facilitación de los datos microbiológicos y del Servicio de Admisión solicitados. A la Dra. A.García por la revisión crítica del manuscrito. 


\section{Bibliografía}

1. Control of tuberculosis in the United States. Am Rev Respir Dis 1992; 146: $1623-33$.

2. Guidelines for preventing transmission of Mycobacterium tuberculosis in health-care facilities, 1994. Centers for Disease Control and Prevention. MMWR Morb Mortal Wkly Rep. 1994; 43: 1-132.

3. The American Lung Association Conference on re-establishing control of tuberculosis in the United States. Am J Respir Crit Care Med 1996; 154: 251-62.

4. American Thoracic Society; CDC; Infectious Diseases Society of America. Treatment of tuberculosis. MMWR Recomm Rep 2003; 52 (RR11): 1-77.

5. Pablos Méndez A, Sterling TR, Frieden TR, et al. The relationship between delayed or incomplete treatment and all-cause mortality in patients with tuberculosis. JAMA 1996; 276: 1223-8.

6. Pearson ML, Jereb JA, Frieden TR, Crawford JT. Nosocomial transmission of multi-drug resistant Mycobacterium tuberculosis: A risk to patients and health care workers. Ann Intern Med 1992; 117: 191-6.

7. Menzies RI, Fanning A, Yuan L, et al. Tuberculosis among health care workers. N Engl J Med 1995; 332: 92-8.

8. Guerrero A, Cobo J, Fortún J, Navas E, Quereda C, Asensio A, et al. Nosocomial transmisión of Mycobacterium bovis resistant to 11 drugs in people with advanced HIV-1 infection. Lancet 1997; 35: 1738-42.

9. Scott B, Schmid M, Nettleman MD: Early identification and isolation of inpatients at high risk for tuberculosis. Arch Inter Med 1994; 154: 32630 .

10. Mathur P, Sacks L, Auten G, Sall R, Levy C, Gordin F: Delayed diagnosis of pulmonary tuberculosis in city hospitals. Arch Inter Med 1994; 154: 306-10.

11. Moran GJ, McCabe F, Morgan MT, Talan DA: Delayed recognition and infection control for tuberculosis in the emergency department. Ann Emerg Med 1995; 26: 290-6.

12. Venkatarama KR, Iademarco EP, Fraser VJ, Kollef MH. Delays in the suspicion and treatment of tuberculosis among hospitalized patients. Ann Intern Med 1999; 130: 404-11.

13. Greenaway C, D. Menzies, A. Fanning, et al. Delay in Diagnosis among Hospitalized Patients with Active Tuberculosis, Predictors and Outcomes. Am J Respir Crit Care Med 2002; 165: 927-33.

14. J.L. Mostaza, S. Fernández, A. Bahamonde, et al. Epidemiología de la tuberculosis en el Bierzo. IX Congreso Nacional de Enfermedades Infecciosas y Microbiología Clínica. 2000. Enferm Infecc Microbiol Clin 2000; 18 (Supl. 1): 123.

15. Martin V, Aránzazu Alonso M, Ramos J, Otero A, Cortizo J, Travieso $\mathrm{S}$. The incidence of respiratory tuberculosis in the province of Leon according to the system of notification of disease mandatory declaration, 1992-1999. Rev Esp Salud Publica 2002; 76: 239-48.

16. Casal M, Gutiérrez Aroca J, Jiménez, Rubio R. Estudio del retraso de la detección y tratamientos en casos de Tuberculosis. Rev Clin Esp 1986; 178: 109-11.

17. Franco J, Blanquer R, Flores J. Fernández E. Plaza P. Nogueira JM. Análisis del retraso diagnóstico en la tuberculosis. Med Clin (Barc) 1996; 107; 453-7.
18. Grupo de trabajo de PMIT . Diagnóstico y tratamiento de la tuberculosis en España. Resultados del Proyecto Multicéntrico de Investigación en Tuberculosis (PMIT). Med Clin (Barc) 2001; 116: 167-73.

19. Altet Gómez MN, Alcaide Megías J, Canela Soler J, Milá Augé C, Jiménez Fuentes MA, De Souza Galvao ML, et al. Estudio del retraso diagnóstico de la tuberculosis pulmonar sintomática. Arch Bronconeumol 2003; 39: 146-52.

20. Mostaza JL, Bahamonde A. Retraso en el diagnóstico y tratamiento de pacientes hospitalizados con tuberculosis. Enferm Infec Microbiol Clin 2004; 22: 59-60.

21. Mostaza JL, Fuentes MI, Palomo MJ, Garcia N. Impacto de una intervención educativa simple en el retraso diagnóstico de los pacientes hospitalizados con tuberculosis pulmonar. Med Clin (Barc) 2005; 124: 315.

22. Controlling Tuberculosis in the United States. Recommendations from the American Thoracic Society, CDC, and the Infectious Diseases Society of America. MMWR 2005; 54 (RR12); 1-81.

23. Behr MA, Warren SA, Salamon H, et al. Transmission of Mycobacterium tuberculosis from patients smear-negative for acid-fast bacilli. Lancet 1999; 353: 444-9.

24. Wisnivesky JP, Kaplan J, Henschke C, McGinn TG, Crystal RG. Evaluation of Clinical Parameters to Predict Mycobacterium tuberculosis in Inpatients. Arch Intern Med 2000; 160: 2471-6.

25. Cobo J, Oliva J, Asensio A, Navas E, Cobo ME, Sánchez-García MD, et al. Predicting Tuberculosis Among HIV-Infected Patients Admitted to Hospital: Comparison of a Model with Clinical Judgment of Infectious Disease Specialists. Eur J Clin Microbiol Infect Dis 2001; 20: $779-84$

26. Moreno S, Cobo J. Las múltiples caras del control de la tuberculosis. Enferm Infecc Microbiol Clin.2003; 21: 279-80.

27. Mosquera JA, Rodrigo L, Gonzálvez F. The evolution of pulmonary tuberculosis in coal miners in Asturias, northern Spain. An attempt to reduce the rate over a 15-year period, 1971-1985. Eur J Epidemiol 1994; 10: 291-7.

28. Chang $\mathrm{KC}$, Leung CC, Tam CM. Tuberculosis risk factors in a silicotic cohort in Hong Kong. Int J Tuberc Lung Dis 2001; 5: 177-84.

29. Barnes PF, Bloch A, Davidson PT, Snider DJ: Tuberculosis in patients with human immunodeficiency virus infection. N Eng J Med 1991; 324: 1644-50.

30. Keane J, Gershon S, Wise RP, Mirabile-Levens E, Kasznica J, Schwieterman WD, et al. Tuberculosis associated with infliximab, a tumor necrosis factor alpha-neutralizing agent. N Engl J Med 2001; 345: 1098-104.

31. Bass JB Jr, Farer LS, Hopewell PC, O'Brien R, Jacobs RF, Ruben F, et al. Treatment of tuberculosis and tuberculous infection in adults and children. Am J Respir Crit Care Med 1994; 149: 1359-7.

32. Neff M; ATS; CDC; IDSA. ATS, CDC, and IDSA update recommendations on the treatment of tuberculosis. Am Fam Physician 2003; 68: 1854, 1857-8, 1861-2.

33. Gordin FM, Slutkin G, Schecter G, Goodman PC, Hopewell PC. Presumptive diagnosis and treatment of pulmonary tuberculosis based on radiographic findings. Am Rev Respir Dis 1989; 139: 1090-3. 